\title{
Burkholderia sp. from rhizosphere of Rhododendron arboretum: Isolation, identification and plant growth promotory (PGP) activities
}

\author{
Shweta Nailwal, Md. Shahbaz Anwar*, Kamal Kant Budhani, Amit Verma ${ }^{1}$, Tapan \\ Kumar Nailwal
}

Department of Biotechnology, Kumaun University Nainital, Bhimtal Campus, Bhimtal-263136 (Uttarakhand), INDIA

${ }^{1}$ Department of Biochemistry, GBPUA and T, Pantnagar- 263145 (Uttarakhand), INDIA

*Corresponding author. E-mail: shahbazmicro@gmail.com

Received: May 18, 2014; Revised received: August 22, 2014 ; Accepted: October 10, 2014

\begin{abstract}
Plant growth promoting rhizobacteria (PGPR) is beneficial bacteria that colonize plant roots and enhance plant growth by wide variety of mechanism like phosphate solubilisation, etc. Use of PGPR has steadily increased in agriculture and offers an attractive way to replace chemical fertilizers, pesticides and supplements. The present research work was designed to isolate and characterize the PGP activity of Burkholderia sp. For this purpose rhizospheric soil from Rhododendron arboreum of Kumaun Himalaya was collected and efficient bacterial strain was screened on the basis of phosphate solubilization. Further, assessment of various parameters of plant growth

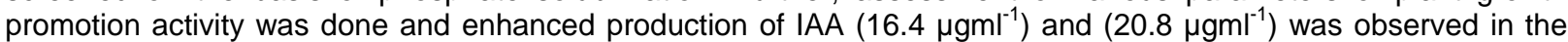
presence of $250 \mathrm{\mu gml}^{-1}$ and $500 \mathrm{~g} \mathrm{mll}^{-1}$ of tryptophan, respectively. Correspondingly, in respect of $7.8 \mu \mathrm{g} \mathrm{ml} l^{-1} \mathrm{IAA}$ without tryptophan, and their confirmation was executed by TLC. A remarkable change in color from green to reddish-brown zone on CAS plates, suggests the positive result for siderophore production, and finally the seed germination and pot trial experiment depicted the growth index of wheat plant. Therefore, the present study suggests that Burkholderia sp. is beneficial for plant growth promotion.
\end{abstract}

Keyword: Burkholderia sp., IAA, PGPR, Phosphate solubilization, Siderophore

\section{INTRODUCTION}

Plant growth promoting rhizobacteria (PGPR) are group of bacteria that actively colonize plant roots and increase plant growth and yield. The mechanism by which PGPR promote plant growth is not fully understood, but it is thought to include the ability to produce phytohormones, asymbiotic $\mathrm{N}$ fixation against phytopathogenic microorganisms by production of siderophores, synthesis of antibiotics, enzymes and fungicidal compounds (Ahmad et al., 2006). In this connection the beneficial, root-colonizing, PGPR are defined by three intrinsic characteristics: (i) they must be able to colonize the root, (ii) they must survive and multiply in microhabitats associated with the root surface, in competition with other micro biota, at least for the time needed to express their plant promotion/protection activities, and (iii) they must promote plant growth. Several novel techniques to identify and characterize the PGPR and to study the colonization pattern and molecular determinants of root colonization have been extensively discussed (Gamalero et al., 2004). PGPR can affect plant growth by different direct and indirect mechanisms (Naz et al., 2012) like enhanced resistance to pathogenic diseases (Shobha and Kumudini, 2012). Direct methods of increasing plant growth are through production of phytohormones, such as auxin, cytokinin and gibberellin. Screening PGPR isolates for quantity of auxin production has been proposed as a mechanism for selecting PGPR species for wheat inoculation (Khalid et al., 2004). These studies indicate that higher the plant growth promoting activities of rhizobacteria, higher is the chance of increased plant growth under different conditions. Hence, isolation and characterization of strains with higher plant growth promoting abilities, adapted under certain climates, can be very useful for the production of effective biofertilizers (Dastager et al., 2010; Abbas et al., 2010). A number of different bacteria promote plant growth, including Azotobacter sp., Azospirillum sp., Pseudomonas sp., Bacillus sp. and Acetobacter sp. (Turan et al., 2006). Plant growth promoting bacteria are important in managing plant growth because of their effects on soil conditions, nutrient availability, growth and yields. Therefore, the aim of this study was to check various PGPR activities of isolated and screened, phosphate solubilizing bacteria. For this purpose the PGP rhizobacteria was isolated as well as screened out and in conclusion characterized on the base of their direct (IAA production) and indirect (Siderophore production) plant growth promoting activities along with the measurement of their growth 
index.

\section{MATERIALS AND METHODS}

Isolation, screening and biochemical characterization of bacterial strain: Bacterial strain was isolated from rhizospheric soil of Rhododendron arboreum of Kumaun Himalayan region. The rhizospheric soil sample were taken from a depth of $0-10 \mathrm{~cm}$, kept in plastic bags and carried to the laboratory. The pure culture was isolated by using serial dilution and streak plate technique to obtain single colony. The culture was screened out on the basis of its ability to solubilize insoluble inorganic phosphate by spotting overnight grown cultures on Pikovskaya's agar plates and incubated at $30^{\circ} \mathrm{C}$. Isolated strain, showed clear zone in the plate around the colony after 48 to 72 hrs of incubation.

Further, the identification of bacterial strain $\mathrm{BS}_{4}$ was carried out according to Bergey's manual on systematic bacteriology (Holt et al., 1994). Catalase activity was determined by detective bubble formation with $3 \%$ $\mathrm{H}_{2} \mathrm{O}_{2}$ solution. Oxidase test was determined by using bacteriological differentiation oxidase disc (Hi-Media Laboratories, India). Other important biochemical properties were performed by using biochemical test kits (KB001, KB002 HiAssorted ${ }^{\mathrm{TM}}$ ).

Molecular characterization: Based on screening and biochemical experiment, potential strain $\mathrm{BS}_{4}$ was characterized on the basis of 16S rRNA sequencing. Polymerase chain reaction (PCR) amplification of the partial 16S rRNA gene region was carried out with the bacterial primer set FD1: 5`-ccgaattcgtcgacaac AGAGTTTGATCCTGGCTCAG-3 and RP2: 5 - c c cgggatccaagctt ACGGCT ACC T T G T TACGACTT-3', using the Gene amp kit (Applied Biosystems). PCR System 9700, U.S.A Pikovaskaya, 1948. About 50 -100 mg of the purified DNA was sequenced using BigDye Terminator Cycle. Sequencing Kits (v3.1) (Applied Biosystems, USA) the templates were purified by Ethanol/EDTA precipitation method and sequenced on ABI 3730xls Genetic Analyzer (Applied Biosystems) at Bioserve Biotechnologies, Pvt. Ltd. Hyderabad, Andhra Pradesh, India. The sequence was then analyzed by basic local alignment search tool (BLAST) at National Center for Biotechnology Information, USA (NCBI) database. Further, the isolate was identified based on the similarity scores. Phylogenetic tree was constructed using the unweighted pair group mean average (UPGMA) tree method and the phylogenetic data were obtained by aligning the different sequences of the 16S rRNA of closely related strains.

Quantitative estimation of phosphate (Pi) and indole-3-acetic acid (IAA) production: The isolated strain $\mathrm{BS}_{4}$ was further examined for its ability to release inorganic phosphate from tricalcium phosphate (TCP) in broth medium. One $\mathrm{ml}$ of overnight culture of the isolate was inoculated to $100 \mathrm{ml}$ of Pikovskaya's broth (Pikovaskaya, 1948). The inoculated flask was incubated at $30^{\circ} \mathrm{C}$. The amount of inorganic phosphate released in the broth was estimated by sampling broth culture at every 24 hours. The broth cultures were centrifuged at $10,000 \mathrm{rpm}$ for 10 minutes to separate the supernatant from the cell growth and insoluble phosphate. The available Phosphate in the supernatant was estimated by phosphomolybdic blue colour method. One $\mathrm{ml}$ of the culture supernatant was taken in a $50 \mathrm{ml}$ volumetric flask to which $10 \mathrm{ml}$ of chloromolybdic acid was added and mixed thoroughly. The volume was made up to approximately three fourth with distilled water and $0.25 \mathrm{ml}$ chlorostannous acid was added to it. Immediately, the volume was made to $50 \mathrm{ml}$ with distilled water and mixed thoroughly. After 15 minutes, the blue colour developed was read in a spectrophotometer at $610 \mathrm{~nm}$ using a reagent blank. Concurrently, a standard curve was prepared using various concentrations of phosphate solution. The amount of phosphorus solubilized by the strain $\mathrm{BS}_{4}$ was calculated using the standard curve.

Quantification of IAA, by strain $\mathrm{BS}_{4}$ was done by inoculating three $250 \mathrm{ml}$ conical flasks containing 100 $\mathrm{ml}$ of minimal salt medium each, supplemented with tryptophan at a concentration of $500 \mu \mathrm{g} / \mathrm{ml}, 250 \mu \mathrm{g} / \mathrm{ml}$ and control without tryptophan, and incubated at $30^{\circ} \mathrm{C}$ under shaking. The amounts of IAA produce in the broth of all flasks were estimated by sampling broth culture after every 24 hours. Broth culture was centrifuged at $7500 \mathrm{rpm}$ for 10 minutes. Two $\mathrm{ml}$ of aliquot of the supernatant of the cultures was mixed with two drops of orthophosphoric acid and $4 \mathrm{~mL}$ of Salkowski reagent $(50 \mathrm{ml}, 35 \%$ perchloric acid; $1 \mathrm{ml}$ $0.5 \mathrm{M} \mathrm{FeCl}_{3}$ ) (Noori and Saud, 2012) and incubated at $30^{\circ} \mathrm{C}$ for 25 minutes. Absorption was read at 530 $\mathrm{nm}$ and the concentration of IAA in the bacterial strain was determined and quantified by comparison with a standard curve of IAA (Reddy et al., 2011).

Extraction of crude IAA and thin layer chromatography (TLC): Single bacterial colony of strain $\mathrm{BS}_{4}$ was inoculated in $100 \mathrm{ml}$ nutrient broth supplemented with $5 \mathrm{mg} / \mathrm{ml}$ tryptophan and incubated at $30^{\circ} \mathrm{C}$ under shaking. Bacterial culture were centrifuged at $7500 \mathrm{rpm}$ for 10 minutes to separate the supernatant and subsequently acidified to $\mathrm{pH} 3$ with $1 \mathrm{~N}$ HCL. Extraction was done twice with ethyl acetate at double the volume of supernatant. Extracted ethyl acetate fraction was evaporated to dryness in a rotary evaporator at $40^{\circ} \mathrm{C}$. The extract was dissolve in $0.5 \mathrm{ml}$ methanol and kept it at $-20^{\circ} \mathrm{C}$. TLC of extracted ethyl acetate fraction (10-30 $\mu \mathrm{l})$ was done and developed in chloroform: ethyl acetate: formic acid (5:3:2). Spot with RF value identical to standard IAA were identified under UV light $(254 \mathrm{~nm})$ by spraying the plates with salkowski reagent.

Seed germination test and pot experiment: Effect of IAA produced by strain $\mathrm{BS}_{4}$ on the growth of plant 
was also studied by seed germination test (Strom and Garhardson, 1988) and pot experiment (Kravchenko, 2004). Wheat (Triticum aestivum) seeds were surface sterilized by exposing to $95 \%$ ethanol and immersing in $0.2 \% \mathrm{HgCl}_{2}$ solution for $3 \mathrm{~min}$. The seeds were then subjected to five times washing with sterile distilled water. One $\mathrm{ml}$ of overnight grown bacterial culture $\left(10^{7}-10^{6}\right.$ cells/ml, 0.6 of O.D at $\left.540 \mathrm{~nm}\right)$ was applied on each seed for $10 \mathrm{~min}$ and treated seeds were dried. For seed germination test the sterile non treated dried seeds as control, soaked with non inoculated media for $10 \mathrm{~min}$ and the treated dried seeds were sown on soft agar $(0.8 \%)$ plates under axenic condition and incubated them at $30^{\circ} \mathrm{C}$ for 5 days. The percent seed germination and root length were measured. For Pot experiment, soil sample were collected, air dried, sieved and sterilized three times repeatedly by autoclaving before filling the pots. Finally, the sterile non treated dried seeds as control, soaked with non inoculated media and the treated dried seeds were transfer to pots containing sterile soil to a depth of $5 \mathrm{~mm}$, the experiment was performed in triplicates. The pots were kept in green house for 15 days under daily observation. After 15 days the plant were uprooted carefully and the length of root and shoot were measured.

Detection of siderophore production: The analysis of siderophore production by the bacterial strain $\mathrm{BS}_{4}$ was performed by Chrome Azurol S (CAS) method. The CAS plates were used to check for the presence of siderophore. The CAS assay is the universal chemical assay for the detection of siderophores. It is based on the high affinity of siderophores for ferric iron, where by ferric iron bound to dye, is released from the dye. The blue color of the medium is due to the dye iron complex. When siderophore is added, the siderophore binds to the ferric iron, releasing the free dye, which is orange in colour.

$\mathrm{Fe} 3+$-dye (blue) + siderophore

$\mathrm{Fe} 3+$-siderophore + dye ( orange)

Hence, the presence of siderophore is indicated by a color change from blue to orange.

The CAS plates were prepared in three separate steps.

(1) Preparation of CAS indicator solution.

(2) Preparation of basal agar medium.

(3) Preparation of CAS agar plates.

Preparation of CAS indicator solution: Initially, $60.5 \mathrm{mg}$ of chrome azurol S was dissolved in $50 \mathrm{ml}$ of $\mathrm{ddH}_{2} \mathrm{O}$. $10 \mathrm{ml}$ of Ferric ion solution $\left(27 \mathrm{mg} \mathrm{FeCl}_{3}\right.$.

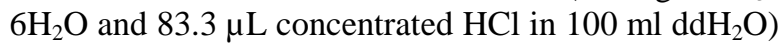
was added, along with $72.9 \mathrm{mg}$ hexadecyltrimethyl ammonium bromide (HDTMA) dissolved in $40 \mathrm{ml}$ $\mathrm{ddH}_{2} \mathrm{O}$. The HDTMA solution was added slowly while stirring, resulting in a dark blue solution $(100 \mathrm{ml}$ total volume), which was then autoclaved.

Preparation of basal agar medium: In a $250 \mathrm{ml}$ flask, 3 gm 3-(N-morpholino) propane sulfonic acid (MOPS) (0.1 M), $0.05 \mathrm{gm} \mathrm{NaCl}, 0.03$ gm $\mathrm{KH}_{2} \mathrm{PO}_{4}, 0.01 \mathrm{~g}$ ammonium chloride $\left(\mathrm{NH}_{3} \mathrm{Cl}\right)$, and 0.05 gm

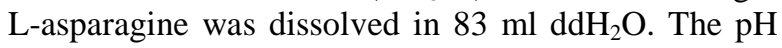
of the solution was adjusted to 6.8 using $6 \mathrm{M} \mathrm{NaOH}$. The total volume was brought to $88 \mathrm{ml}$ using $\mathrm{RO}$ water, and $1.5 \mathrm{~g}$ agar was added to the solution while stirring and heating until melted. The solution was then autoclaved.

Preparation of CAS agar plate: The autoclaved basal agar medium was cooled to $50^{\circ} \mathrm{C}$ in a water bath. The CAS indicator solution was also cooled to $50^{\circ} \mathrm{C}$, along with a $50 \%$ solution of glucose. Once cooled, $2 \mathrm{ml}$ of the $50 \%$ glucose solution was added to the basal agar medium with constant stirring, followed by $10 \mathrm{ml}$ of the CAS indicator solution, which was added carefully and slowly along the walls of the flask with constant stirring, but at a speed so as not to generate any bubbles. Once mixed thoroughly, the resulting solution $(100 \mathrm{ml})$ was poured into sterile plastic plates, each plate receiving approximately $25 \mathrm{ml}$ of blue agar. Under minimal iron conditions, siderophore is produced and released into the culture medium. To detect the siderophore production strain $\mathrm{BS}_{4}$ was grown in iron-restricted $(0.5 \mu \mathrm{M}$ added iron $)$ modified minimal medium. After 24 hours of growth, the culture was inoculated to CAS plates and the plate was incubated at $30^{\circ} \mathrm{C}$ temperature to develop. The production of siderophore was confirmed by the presence of an orange colour halo zone around the colony.

\section{RESULTS AND DISCUSSION}

PGPR colonize plant roots and exert beneficial effects on plant growth and development by a wide variety of mechanisms. The present study deals with the isolation and characterization of various plant growths promoting activity of strain $\mathrm{BS}_{4}$, to execute this, the strain has been isolated, screened and assessed for various parameters indicative of both the direct and indirect mechanisms of plant growth promotion along with wheat growth index by pot trial experiment.

Bacterial isolation and screening: For bacterial isolation, soil sample were collected from rhizospheric soil of Rhododendron arboreum of Kumaun Himalayan region. Bacterial strain $\mathrm{BS}_{4}$ was isolated from the soil sample on nutrient agar media and screen out on the basis of high phosphate solubilisation activity on Pikovaskya's agar plate (Fig. 1A). The positive result was determined by halo zone formation around the bacterial colony on the Pikovaskya's agar plate.

Biochemical characterization: Cellular morphology of screened bacterial strain was studied by performing gram staining and observing cells under light microscope. Biochemical characterization of the strain was done by different tests like catalase, nitrate reductase, dextrose, sucrose fermentation, voges pouskar test, oxidase test, $\mathrm{H}_{2} \mathrm{~S}$ production and methyl red test (Table 1). Attractively, the strain responded differently towards the entire biochemical test, this behavior suggests their 

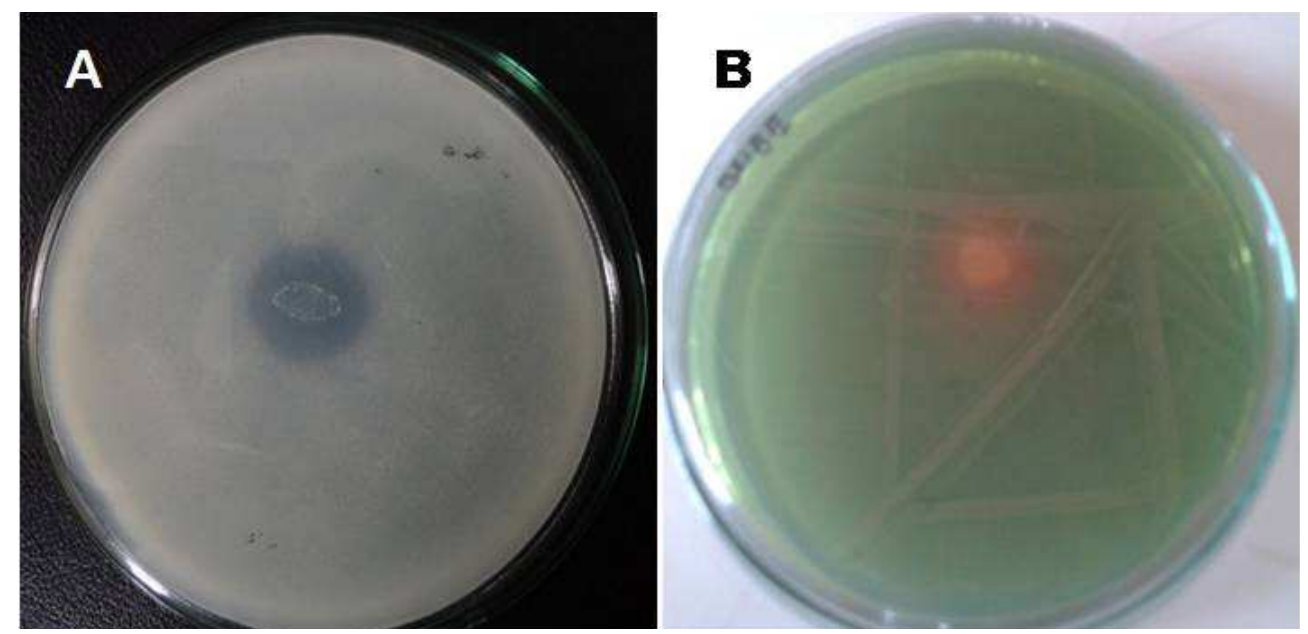

Fig.1. Phosphate solubilization and Siderophore production: A- The zone of inorganic phosphate solubilised (halo zone) on Pikovskaya's agar plate. B- The siderophore produced (orange colour zone) from bacterial culture on CAS agar plate).

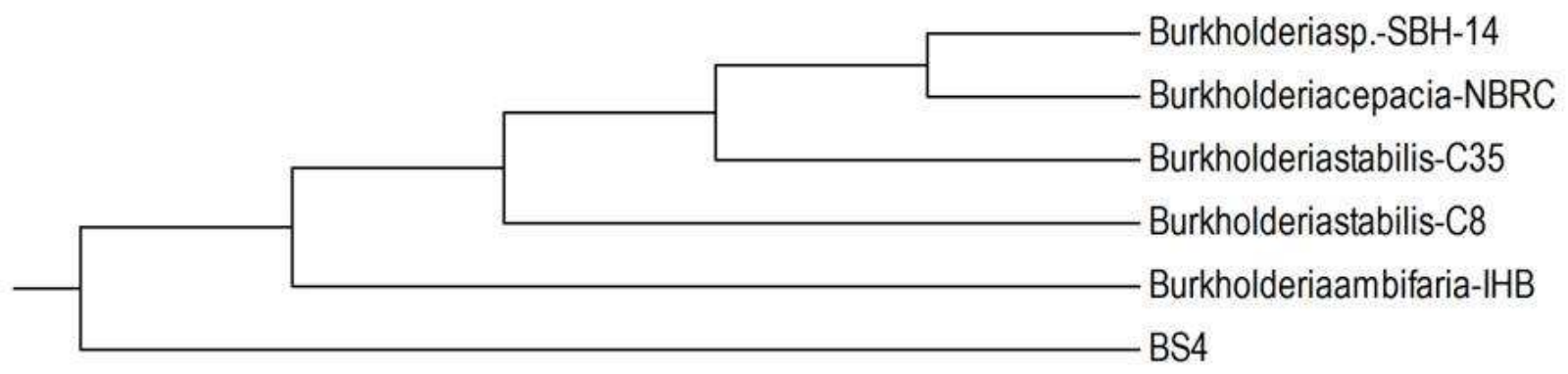

Fig. 2. Phylogenetic tree for the $16 S$ rRNA sequences of the bacterial strain BS4 constructed by using MEGA4 software by UPGMA method.
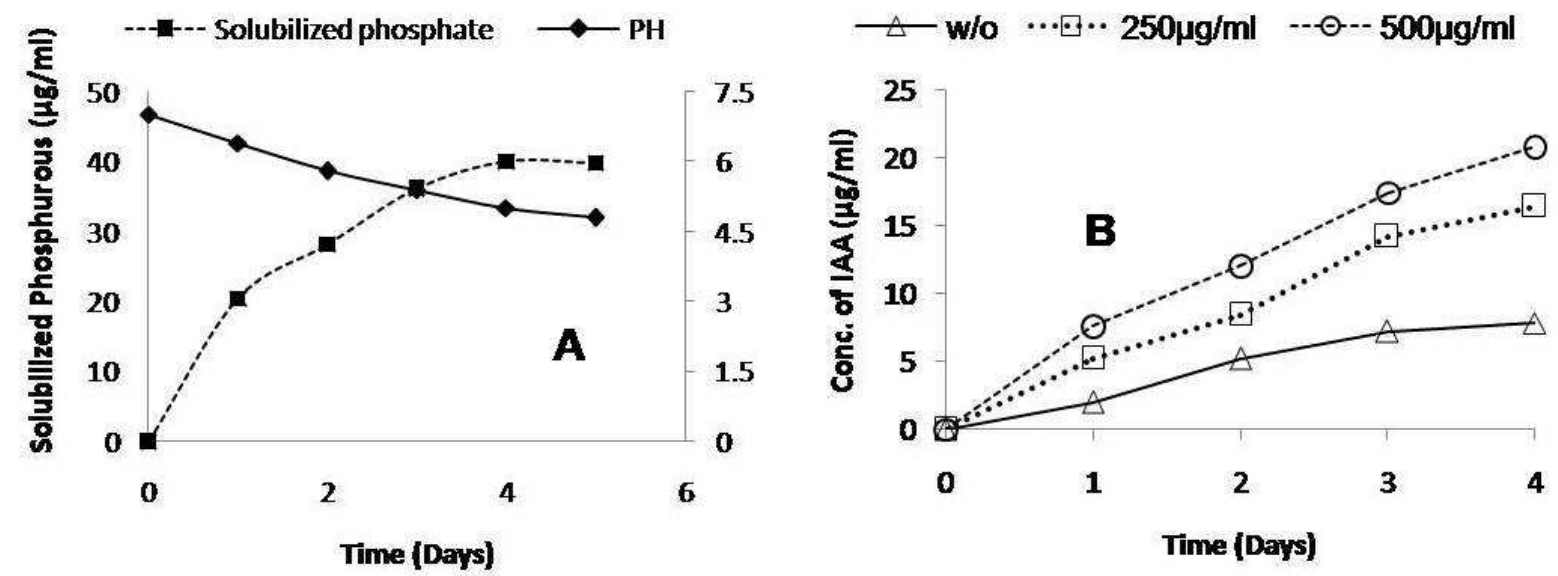

Fig. 3. Assessment of phosphate solubilizing and IAA producing activity of the strain BS4 as a function of time. A- The concentration IAA on divergent level of tryptophan. $B$ - The amount of soluble phosphate determined from the absorbance data using the calibration curve with $\mathrm{KH}_{2} \mathrm{PO}_{4}$ at $600 \mathrm{~nm}$. The right-axis indicates the change in pH of the Pikovskaya's medium during growth.

unique metabolic potential and categorized them putatively into the group of Burkholderia.

Molecular characterization and analysis phylogenetic tree: Selected strain $\mathrm{BS}_{4}$ was subjected to $16 \mathrm{~S}$ rRNA sequencing and was analyzed using different bioinformatics tools. The partial sequence data of the isolates was analyzed by BLAST search that showed unambiguous similarity (98-99\%) with Burkholderia sp. Further, the phylogenetic tree was constructed using MEGA4 software by neighbour-joining tree method and the phylogenetic data were obtained by aligning the different sequences of the 16S rRNA of closely related strains (Fig. 2).

Siderophore production and quantification of solubilized phosphate: Siderophore production of by Burkholderia sp. was confirmed by CAS assay. 
Table 1. Morphological and biochemical characteristics of bacterial strain BS4.

\begin{tabular}{|c|c|c|}
\hline S. $\mathbf{N}$. & Biochemical Test & Response \\
\hline 1. & Gram Staining & Gram negative \\
\hline 2. & Cellular morphology & Rod shape \\
\hline 3. & Citrate Utilization & Positive \\
\hline 4. & Lysine Utilization & Positive \\
\hline 5. & Ornithine Utilization & Positive \\
\hline 6. & Urease & Negative \\
\hline 7. & Phenylalanine Deaminase & Negative \\
\hline 8. & Nitrate Reduction & Positive \\
\hline 9. & $\mathrm{H}_{2} \mathrm{~S}$ Production & Negative \\
\hline 10. & Catalase Test & Positive \\
\hline 11. & Lactose & Positive \\
\hline 12. & Xylose & Positive \\
\hline 13. & Maltose & Positive \\
\hline 14. & Fructose & Positive \\
\hline 15. & Dextrose & Positive \\
\hline 16. & Galactose & Positive \\
\hline 17. & Raffinose & Negative \\
\hline 18. & Trehalose & Negative \\
\hline 19. & Melibiose & Negative \\
\hline 20. & Sucrose & Negative \\
\hline 21. & L-Arabinose & Negative \\
\hline 22. & Mannose & Positive \\
\hline 23. & Adonitol & Negative \\
\hline 24. & Sorbitol & Negative \\
\hline 25. & Glucose & Positive \\
\hline 26. & Oxidase & Positive \\
\hline
\end{tabular}

Appearance of a reddish-brown zone on CAS plates suggests the positive result for siderophore production (Fig.1B). This low molecular weight iron binding protein is well known to exhibit antagonistic activity against phytopathogenic fungi. Involvement of the siderophore in the suppression of Pythium-induced damping-off of tomato by $P$. aeruginosa TNS K2 has been demonstrated using pyoverdine-deficient mutants (Buysens et al., 1996). Similar increase in the biocontrol potential of the siderophore (Bakthavatchalu et al., 2012) over producing mutant MPS 16 M-1 of Pseudomonas sp. against Rhizoctonia solani in chickpea has also been reported (Goel et al., 2002). Comparable finding related to siderophore production by different bacterial strains were documented by Sakthivel and Karthikeyan ( 2012).

Although, The efficiency of Burkholderia sp. was carried out in Pikovskaya's broth and amount of soluble phosphate released in liquid medium due to solubilization of tri-calcium phosphate has been determined using the calibration curve of $\mathrm{KH}_{2} \mathrm{PO}_{4}$ at $600 \mathrm{~nm}$ to be $\left(40.0 \mathrm{mgml}^{-1}\right)$ upon 5 days of growth. The data showed the time-dependent increase in the amount of solubilised phosphate, and inverse relationship with the $\mathrm{pH}$ of the medium. Periodic monitoring of $\mathrm{pH}$ of the culture filtrate revealed a significant reduction from $\mathrm{pH} 7.0$ to 4.6 (Fig. 3B). The relationship of decrease in $\mathrm{pH}$ of the culture medium with the increase amount of phosphate solubilization is well recognized (Kumar et al., 2012; Anwar et al., 2014). Thus, the results suggest the enhanced phosphate solubilization activity with the screened bacterial strain could be due to result of higher acid production. The production of organic acid and its role in phosphate solubilization is well known, reported in earlier (Kaur and Sharma, 2013; Sujatha and Ammani, 2014).

IAA Production, quantification and confirmation with TLC: Estimation of IAA produced by Burkholderia sp. was carried out using the calibration curve of IAA. The extended incubation of culture up to 4 days showed some reduction in cell viability. Fig. 4 A, clearly demonstrates the production of significant amount of IAA in LB broth medium. However, sufficient bacterial biomass persisted in the stationary phase culture

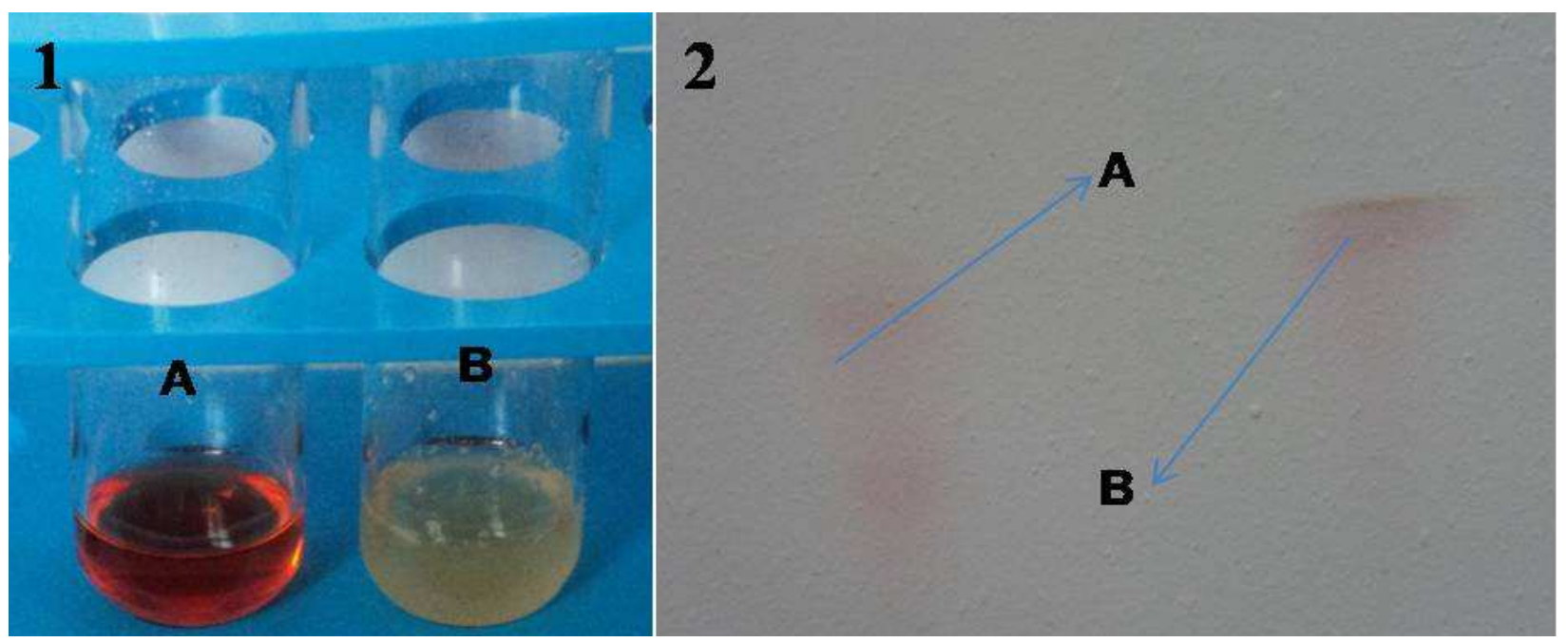

Fig. 4. Estimation and confirmation of IAA production: 1 - Positive result of IAA production $(B)$ with compare with negative control (A): 2- TLC of indole-3-acetic acid detected by Salkowiski reagent; A) Sample, B) Standard. 

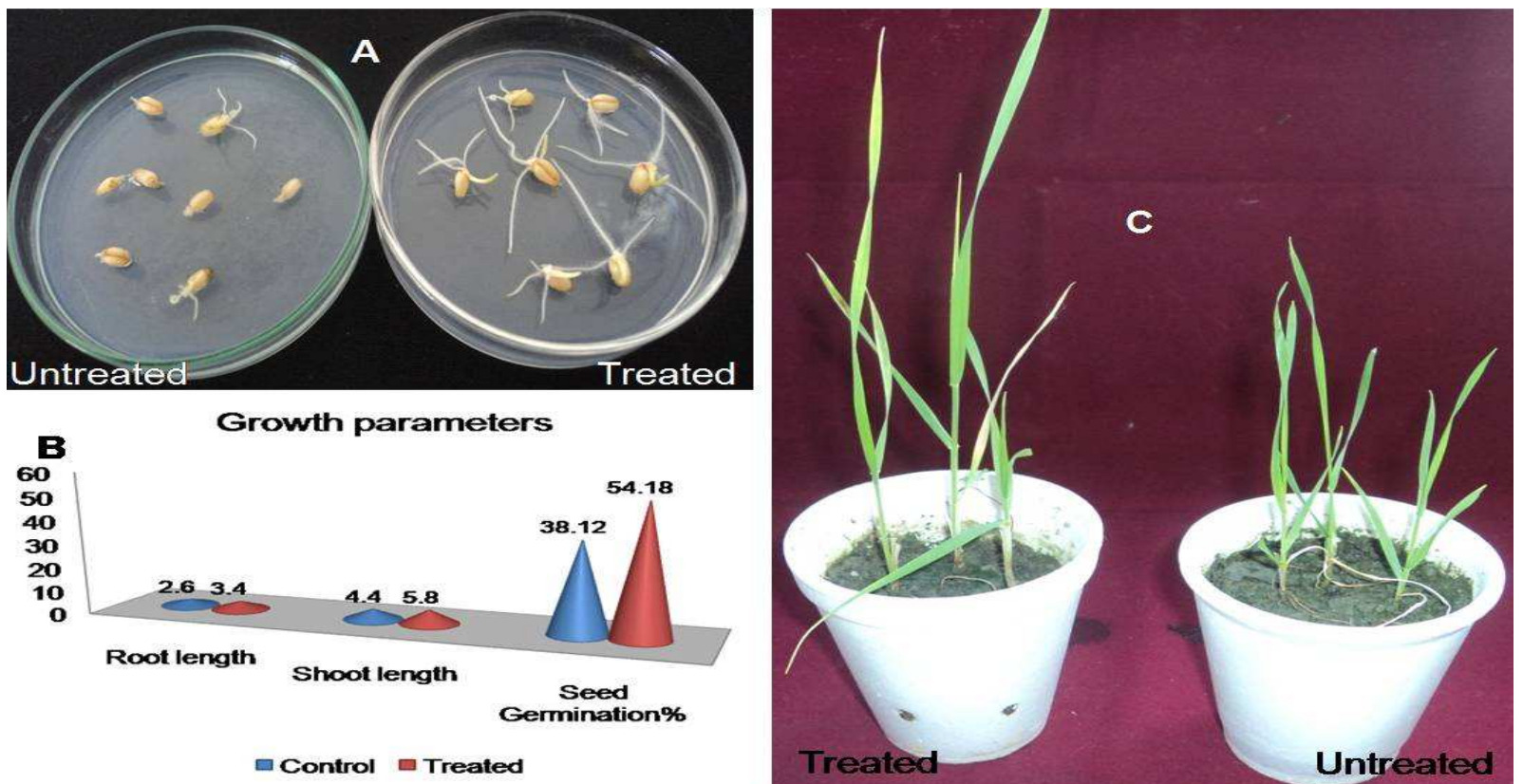

Fig. 5. Evaluation of plant growth promoting activity (A) Seed germination (B) Growth index (C) Shoot/root length

even on $4^{\text {th }}$ day of incubation. The culture filtrate at different time intervals showed a linear and time dependent increase in IAA production. Enhanced production of IAA $\left(16.4 \mu \mathrm{gml}^{-1}\right)$ and $\left(20.8 \mathrm{gml}^{-1}\right)$ was observed in the presence of $250 \mu \mathrm{gml}^{-1}$ and $500 \mu \mathrm{g} \mathrm{ml}^{-1}$ tryptophan, respectively, and $7.8 \mu \mathrm{g} \mathrm{ml}^{-1}$ IAA without tryptophan (Fig. 3A) and similar findings related to IAA quantification were documented by Sakthivel and Karthikeyan (2012). Furthermore, linear increase in IAA production up to $4^{\text {th }}$ day exhibited stability of the metabolic cells during stationary phase. These results correspond with the earlier observations indicating IAA production in stationary phase of culture (Rani and Reddy, 2012). Confirmation of IAA production was done by TLC method (Fig. 4B), in which the culture filtrate of Burkholderia sp. was used to extract IAA for characterization. The spots of ethyl acetate extract of culture and standard IAA were tested in the solvent mixture of chloroform: ethyl acetate: formic acid (5:3:2). Chromatograms of spots were sprayed with salkowski reagent that shows almost the same RF value (0.85). The findings of TLC on the basis of RF value are in agreement with reports by other scientist (Ahmad et al., 2005; Prasanna et al., 2010; Sahasrabudhe, 2011).

Seed germination test and pot experiment: The evaluation of effect on plant growth parameter of these IAA producing Burkholderia sp. were further studied in seed germination and pot experiment (Fig.5). In seeds germination test, the percentage seed germination of treated seed were found high (54.18\%) as compared to control (38.12\%) (Fig.5B). Maximum effect on root length was observed in germinated seed compared to control seed (Fig. 5A), the strain also shows greater length of root and shoot in the harvested plant as compared to control (Fig. 5C). The data obtained from seed germination and pot experiment demonstrated positive effect on root and shoot elongation compared to control. This indicates that the Burkholderia sp. can improve the plant growth development and thus considered as an effective PGPR. Similar type of results was reported by Bharucha et al. (2013), Sivakumar et al. (2012) and Mia et al. (2012).

\section{Conclusion}

Many bacteria strains present in rhizospheric region have been shown to possess plant growth promoting property and plant productivity enhancing activity due to which they are collectively designated as plant growth promoting rhizobacteria (PGPR). PGPR have several characteristics which help them to promote the growth and yield of plant. As elucidated by Ahmad et al. (2006) that to establish a bacterial culture as PGPR, they should have some characteristics like the ability to produce phytohormones, asymbiotic $\mathrm{N}$ fixation against phytopathogenic microorganisms by production of siderophores, synthesis of antibiotics, enzymes and fungicidal compounds. So, in the present study, a bacterial isolate Burkholderia sp. was isolated from Rhododendron arboreum rhizosphere of Kumaun region and characterized for the PGP activity. On the parameters of phosphate solubilisation, IAA production, siderophore production, PGP activity of the present strain was established which was further elaborated by evaluating the effect of bacterial culture on seed germination and growth index of wheat plant in a pot experiment. Hence, the present isolate can act as potential candidates for the development of bio-inoculants for crop plants.

\section{ACKNOWLEDGEMENT}

This work is partially supported by UGC grant to 
Tapan Kumar Nailwal. Senior author (Md. Shahbaz Anwar) also acknowledges Biotechnology Department, Campus Bhimtal, Kumaun University, Nainital Uttarakhand, providing support for completion the work with financial assistance.

\section{REFERENCES}

Abbas, P. Z., Rastin, N. S., Rahmani, H. A., Khavazi, K. and Soltani, A. (2010). Plant growth promoting activities of fluorescent Pseudomonas, isolated from the Iranian soils. Acta. Physiol. Plant, 30: 281-288.

Ahmad, F., Ahmad, I. and Khan, M. S. (2005). Indole acetic acid production by the indigenous isolates of azotobacter and fluorescent Pseudomonas in the presence and absence of tryptophan. Turk. J. Biol., 29: 29-34.

Ahmad, F., Ahmad, I. and Khan, M. S. (2006). Screening of free-living rhizospheric bacteria for their multiple plant growth promoting activities. Microb. Res., 36: 1-9.

Anwar, S. M., Siddique, M. T., Verma, A., Rao, Y., Nailwal, T., Ansari, M. W. and Pande, V. (2014). Multitrait plant growth promoting (PGP) rhizobacterial isolates from Brassica juncea rhizosphere keratin degradation and growth promotion. Comm. Integ. Biol., 7( I): e27683-1-9

Bakthavatchalu, S., Shivakumar, S. and Sullia, S. B. (2012). Identification of multi-trait PGPR isolates and evaluation of their potential as biocontrol agents. Acta Biologica Indica., 1(1): 61-67

Bharucha, U., Patel, K. and Trivedi, U. B. (2013). Optimization of indole acetic acid production by Pseudomonas putida UB1 and its effect as plant growth-promoting rhizobacteria on mustard (Brassica nigra). Agric. Res., 2: 215-221.

Buysens, S., Heungens, K., Poppe, J. N. and Hofte, M. (1996). Involvement of pyochelin and pyoverdin in suppression of pythium-induced damping-off of tomato by Pseudomonas aeruginosa 7NSK2. Appl. Environ. Microbiol., 62(3): 865-871.

Dastager, S. G., Deepa, C. K. and Pandey, A. (2010). Isolation and characterization of novel plant growth promoting Micrococcus sp NII-0909 and its interaction with cowpea. Plant Physiol. Biochem., 48(12): 987-992.

Gamalero, E., Lingua, G., Capri, F. G., Fusconi, A. and Berta, G. (2004). Colonization pattern of primary tomato roots by Pseudomonas fluorescens A6RI characterized by dilution plating, flow cytometry, fluorescence, confocal and scanning electron microscopy. FEMS Microbiol. Ecol., 48: 79-87.

Goel, A. K., Sindhu, S. S. and Dadarwal, K. R. (2002). Stimulation of nodulation and plant growth of chickpea (Cicer arietinum L.) by Pseudomonas spp. antagonisctic to fungal pathogens. Biol. Fertil. Soil, 36: 391-396.

Holt, J. G., Krieg, N. R. and Sneath, P. H. A. (1994). Bergey's mannual of determinative Bacteriology, $19^{\text {th }}$ edition, Williams \& Wilkins, Baltimore.

Kaur, N. and Sharma, P. (2013). Screening and characterization of native Pseudomonas sp. as plant growth promoting rhizobacteria in chickpea (Cicer arietinum L.) rhizosphere. Afr. J. Microbiol. Res., 7(16): 1465-1474.

Khalid, A., Arshad, M. and Kahir, Z. A. (2004). Screening plant growth-promoting rhizobacteria for improving growth and yield of wheat. Appl. Soil. Ecol., 96: 473480.
Kravchenko, L. V., Azarova, T. S., Makarova, N. M. and Tikhonovich, I. A. (2004). The effect of tryptophane of plant root metabolites on the phytostimulating activity of rhizobacteria. Microibiologia, 73(2): 156-158.

Kumar, A., Kumar, A., Devi, S., Patil, S. and Payal, C. (2012). Isolation, screening and characterization of bacteria from Rhizospheric soils for different plant growth promotion (PGP) activities: an in vitro study. Rec. Res. Sci. Technol., 4(1): 01-05.

Mia, M. A. B., Shamsuddin, Z. H. and Mahmood, M. (2012). Effects of rhizobia and plant growth promoting bacteria inoculation on germination and seedling vigor of lowland rice. Afric. J. Biotechnol., 11 (16): 37583765.

Naz, I., Bano, A., Rehman, B., Pervaiz, S. and Iqbal, M. (2012). Potential of Azotobacter vinelandii Khsr1 as bio-inoculant. Afric. J. Biotechnol., 11(45): 1036810372.

Noori, M. S. S. and Saud, H. M. (2012). Potential plant growth-promoting activity of Pseudomonas sp isolated from paddy soil in Malaysia as biocontrol agent. Plant. Pathol. Microbiol., 3(2): 120.

Pikovaskaya, R. I. (1948). Mobilization of phosphorus in soil in connection with the vital activity of some microbial species. Micobiologia, 17: 362-370.

Prasanna, R., Joshi, M., Rana, A. and Nain, L. (2010). Modulation of IAA production in cyanobacteria by tryptophan and light. Polish J. Microbiol., 59(2): 99105.

Rani, M. U. and Reddy, G. A. (2012). Screening of rhizobacteria containing plant growth promoting (PGPR) traits in rhizosphere soils and their role in enhancing growth of pigeon pea. Afric. J. Biotechnol., 11(32): 8085-8091.

Reddy, V., Rao, K.N. and Reddy, S.M. (2011). Production of IAA by earthworm-borne fungi. Scholars Research Library. Ann. Biol. Res., 2 (3): 178-181.

Sakthivel, U. and Karthikeyan, B. (2012). Isolation and characterization of plant growth promoting rhizobacteria (pgpr) from the rhizosphere of coleus forskohlii grown soil. Int. J. Rec. Sci. Res. 3(5): $288-296$.

Sahasrabudhe, M. M. (2011). Screening of rhizobia for indole acetic acid production, Schol. Res. Lib. Ann. Biol. Res., 2: 460-468.

Shobha, G. and Kumudini, B. S. (2012). Antagonistic effect of the newly isolated PGPR Bacillus spp. on Fusarium oxysporum. Int. J. App. Sci. Engin. Res., 1(3): 463-474.

Sivakumar, T., Shankar, T., Vijayabaskar, P. and Ramasubramanian, V. (2012). Plant growth promoting activity of nickel tolerant Bacillus cereus TS1. J. Agric. Technol., 8: 2101-2113.

Strom, B. and Garhardson, B. (1988). Differential reaction of wheat and Pea genotypes to root inoculation with growth affecting rhizosphere bacteria. Plant. Soil, 109: 263-267.

Sujatha, N. and Ammani, K. (2014). Phosphate solubilisation by the Isolates of Fluorescent pseudomonads. Int. $J$. Adv. Pharm. Boil. Chem., 3(1): 24-28,

Turan, M., Ataoglu, N. and Sahin, F. (2006). Evaluation of the capacity of phosphate solubilizing bacteria and Fungi on the different form of phosphorous in liquid culture. Sustain. Agric., 28: 99-108. 